\title{
La documentación, herramienta básica del periodista y del comunicador
}

\author{
Dra. Ángeles López Hernández \\ Profesora de Documentación Audiovisual, Periodística e Informativa. \\ Departamento de Periodismo. Universidad de Sevilla.
}

\section{RESUMEN}

El conocimiento de las técnicas documentales ha dejado de ser un terreno exclusivo del documentalista o experto en Documentación para convertirse en una de las herramientas más eficaces del profesional de la Información y de la Comunicación. No parece tener ya cabida alguna en nuestra llamada "sociedad de la información» el profesional mal documentado (indocumentado) y desconocedor de las vías por las que circulan y se accede a los conocimientos. En un mundo donde prima la especialización, el periodista indocumentado es visto hoy día como una persona no suficientemente preparada para afrontar con eficacia su trabajo periodístico $y$, por ende, ser útil a los ojos de su medio de comunicación.

\section{ABSTRACT}

The knowledge of documental techniques is not just an exclusive land for an expert. In fact, Documentation is nowdays one of the most effective tools for a Information or Communication professional. In the "society of the information", it is not right to find a not well documented professional that ignore the ways to obtain the required knowledge. That is why an undocumented journalist is seen like a not sufficiently prepared person to confront its journalistic work with effectiveness.

Palabras claves: Documentación/Periodismo/Comunicación/Investigación en información/Búsqueda documenal.

Key Words: Information Science/Journalism/Communication/Information research/Documentary searching.

\section{La documentación al servicio de la investigación en Información}

$\int$ Inmersos como nos encontramos en la sociedad de la información -o más propiamente llamada de la saturación informativa- al periodista le resulta cada vez más necesario conocer las técnicas documentales que le permitan la que termine por controlarle a él.

La Documentación sigue siendo contemplada como una disciplina cuyos contenidos teóricos y prácticos tienen como finalidad exclusiva el perfeccionamiento del proceso documental desarrollado por quienes trabajan en los centros de 
documentación, a fin de mejorar sus técnicas de trabajo y hacer, en consecuencia, más efectiva su labor. Y no cabe duda de que, en buena medida, es así. Uno de los principales objetivos en los que hoy día trabaja la ciencia documental es el de desarrollar nuevas metodologías que permitan el perfeccionamiento paulatino de las técnicas y herramientas empleadas por los documentalistas para la conservación y difusión eficaz de los documentos.

No obstante, hemos de decir que no es ese el único terreno de trabajo de la Documentación. Esta disciplina también centra su estudio en muchos otros aspectos prácticos relacionados con los documentos y su difusión, como puedan ser:

a) La identificación y clasificación de los distintos tipos de centros de documentación existentes hoy en día.

b) La sistematización y conocimiento de las diversas fuentes de información que pueden ser manejadas o consultadas.

Por tanto, la Documentación nos proporciona los conocimientos básicos que precisamos para emprender con eficacia una búsqueda documental: a dónde acudir y qué soportes documentales nos interesa particularmente manejar (libros, revistas, documentos audiovisuales...)

Pero también a través de esta disciplina podemos llegar a conocer las técnicas más apropiadas a seguir en el tratamiento de los diferentes documentos que van pasando a formar parte de nuestro archivo personal. Dicho en otros términos, la Documentación nos enseña a clasificar, organizar, resumir y extraer las palabras claves (indización) de los mensajes de la manera más eficiente y útil, facilitando y agilizando la recuperación de las informaciones en el momento preciso - presente o futuro- en que nos son necesarias para realizar con eficacia, rigor y calidad nuestro trabajo.

En los últimos tiempos está cambiando a pasos agigantados nuestro rol de usuarios de documentos. Hasta hace pocas décadas, la necesidad de documentarse a fondo acerca de una determinada materia parecía estar cubierta con una simple visita a la biblioteca más cercana o a los archivos de nuestra institución (en aquellos casos naturalmente en que la institución propia contara con este servicio), lugares ambos en los que siempre se hallaba la inestimable ayuda del bibliotecario o del archivero, quienes perfilaban nuestras búsquedas informativas y nos proporcionaban directamente el material documental que nos era preciso, o en caso de no poseer el centro los documentos solicitados, nos facilitaban información útil acerca de dónde poder localizarlos.

Hoy en día, la situación ha cambiado radicalmente. Las nuevas tecnologías de la información y la cada día más imperante necesidad de los investigadores y profesionales de la comunicación de estar bien informados (documentados) se han convertido en dos fenómenos estrechamente interrelacionados.

Así, los avances tecnológicos -primero el ordenador con la expansión de las bases y bancos de datos y, después, Internet- han venido a facilitarnos enorme- 
mente las tareas de búsqueda de las fuentes documentales que precisamos, permitiéndonos entrar y consultar en bibliotecas y/o en centros de documentación localizados en cualquier punto del planeta, con escaso coste económico y de tiempo, algo impensable hace unos años.

Si bien las nuevas tecnologías nos acercan los conocimientos, superando las barreras geográficas, también es cierto que generan nuevas necesidades informativas, sintiéndonos cada día más esclavos de la información. No parece tener ya cabida alguna en nuestra llamada sociedad de la información, un individuo mal documentado (indocumentado) y desconocedor de las vías por las que circulan y se accede a los conocimientos. En un mundo donde prima la especialización, una persona indocumentada es hoy día sinónimo de persona no cualificada, no suficientemente preparada para afrontar con eficacia su trabajo y, por ende, ser útil a los ojos de su institución. Como nos dice Payeur ${ }^{1}$ : «Internet es un sistema que creará [yo diría que ha creado ya] nuevas formas de analfabetismo».

En definitiva, estamos pasando de ser simples usuarios de a pie de bibliotecas, archivos y centros de documentación, a convertirnos en auténticos autodocumentalistas, esto es, en individuos que se ven abocados a conseguir por sí mismos aquellos documentos que les son de interés o que constituyen un instrumento básico de su trabajo cotidiano. En palabras de López Yepes, "estamos pasando de ser homo sapiens, necesitados de información, a ser homo documentalis, capaces de autoinformarnos hasta cierto límite"2. Ahora bien, la pregunta que se suscita entonces es la de si los investigadores y profesionales del mundo de la comunicación saben autodocumentarse convenientemente. Saber autodocumentarse supone, en pocas palabras, saber buscar y recuperar las fuentes documentales que se necesitan para cubrir tales necesidades informativas. Detengámonos en este punto.

\section{La estrategia de búsqueda y recuperación de información}

El primer requisito básico para iniciar una búsqueda rápida y eficaz de información es perfilar con la mayor exactitud posible nuestra demanda, esto es, qué buscamos.

La provisión de fuentes se simplifica si ésta se restringe a un único campo de conocimiento. Éste es el caso de las investigaciones encaminadas a la elaboración de una tesis doctoral, siempre tendente al estudio de un tema muy especializado (pongamos como ejemplo una tesis sobre la Historia del Periodismo en la provincia de Sevilla) o, entrando en el terreno de la actividad profesional, el caso

1 Autor citado por CORDÓN GARCÍA, J.A.: "Sobre la información, su necesidad y los modos de acceder a ella". En TORRES RAMÍREZ, I.: Las fuentes de información. Estudios teórico-prácticos, Madrid: Síntesis, 1998. p. 18

2 LÓPEZ YEPES, J.: Los caminos de la información. Cómo buscar, seleccionar y organizar las fuentes de nuestra documentación personal, Madrid: Fragua, 1997.- p. 24 
del informador que trabaja de manera fija y cotidiana en la sección de economía, política nacional, política internacional, cultura, espectáculos, etc.

No obstante, se ha de tener en cuenta que las diversas áreas de información periodística están basadas o dependen sustancialmente de otras materias más generales o específicas, según los casos, de las que se ha de tener también conocimiento, lo que obliga al investigador y al profesional del periodismo a contar con una colección más abarcadora de lo que en principio pudiera plantearse.

Así, el periodista económico deberá estar bien informado, no sólo de lo relacionado estrictamente con su materia (datos más importantes de la economía, relaciones económicas a nivel mundial, etc), sino también de otros ámbitos colindantes con la misma: por ejemplo, la política regional, nacional o internacional. Igualmente, el investigador en Historia del Periodismo deberá documentarse convenientemente acerca del nacimiento de los diarios, su evolución, etc., y también, como es lógico, de los rasgos característicos (políticos, económicos, sociales, culturales...) de la época que investiga.

Por tanto, y ante la imposibilidad de contar con un fondo documental ilimitado, es necesario que cada investigador o profesional de la Información y de la Comunicación pondere detenidamente y concrete con la máxima precisión que le sea posible qué áreas de conocimiento abarcará su colección particular. Dicho de otro modo, el periodista debe tener muy claro cuáles son los temas o las materias sobre las que necesita estar bien informado y, por ende, documentado para desarrollar con total eficacia su trabajo periodístico. Sólo en ese momento estará en condiciones para iniciar su búsqueda y recuperación de los documentos.

Es esta doble labor de búsqueda y recuperación de documentos la que ha sufrido en muy poco tiempo una importante transformación por el advenimiento de nuevos medios de transmisión de información, causa de la saturación informativa en la que hoy día estamos inmersos y que está provocando un grave colapso en los modernos sistemas de recuperación de los documentos.

Dicha saturación o explosión informativa ha sido consecuencia en buena medida del progreso tecnológico vivido en las últimas décadas en el ámbito del almacenamiento automatizado en multitud de bases de datos de los documentos, y también de la invención y popularización de Internet, donde una ingente y variopinta cantidad de información fluye desorganizadamente.

Hablando del espectacular desarrollo que ha tenido lugar en el último cuarto de siglo dentro de la industria de bases y bancos de datos, Daniel ${ }^{3}$ indica, que, según las estadísticas, las bases de datos disponibles públicamente a nivel mundial han pasado de ser cincuenta y dos millones en 1975 a sumar cerca de cinco

3 DANIEL, E.H.: “Quality Control of Documents”, Library Trends, 41 (4), 1993, pp. 644-664. 
billones en los albores de los años noventa. Según este mismo autor, el efecto inmediato de ello ha sido el dramático incremento, a su vez, en el número de problemas concernientes a la calidad de las informaciones proporcionadas por tales bases de datos, problemas que vienen determinados por factores tales como la falta de actualidad y obsolescencia de muchos de los documentos registrados en dichas bases, las duplicaciones de los registros o la inadecuada selección de los documentos, muchos de ellos triviales.

Ante esta situación, es necesario que el profesional de la comunicación sepa activar su actitud crítica, decidiendo qué bases de datos se ajustan mejor a su perfil de búsqueda y, por otro lado, qué documentos, de los proporcionados por dichas bases, responden más claramente a sus necesidades informativas. De lo contrario, se verá obligado a malgastar buena parte de su tiempo y esfuerzo en la búsqueda y recuperación de multitud de documentos que, tras su lectura, comprobará que le son irrelevantes. O, lo que es peor, sufrirá el llamado por Oberman ${ }^{4}$ Síndrome del Cereal, refiriéndose con ello el autor a la tendencia habitual de los individuos a abandonar la búsqueda cuando les resulta verdaderamente costoso hallar entre el cúmulo inabarcable de documentos que se le ofertan sólo aquéllos que le son útiles.

Asimismo, un estudio realizado por Wiberley y Daugherty ${ }^{5}$ llegaba a la conclusión de que el exceso de información genera en los buscadores un acentuado cansancio intelectual, hasta el punto de que si las referencias recuperadas superan con creces el número de documentos que el usuario está dispuesto a examinar (los autores calculan entre treinta y setenta aproximadamente) se tiende a no recuperarlas y a abandonar de manera definitiva la búsqueda.

Otros muchos investigadores han abordado también este problema de la saturación informativa y las dificultades que encierra, para quien desconoce de las técnicas documentales, el buscar y recuperar selectivamente la información que requiere. Así, Meyer y Ruiz ${ }^{6}$ demostraron la eficacia de fijar en el servicio de bases de datos EasyNet, comercializado por Telebase, un criterio selectivo que serviría al buscador de información para guiarle en su toma de decisiones. Dicho criterio selectivo se denominó "Indicador de recomendación" y se representaba mediante un símbolo que aparecía junto al nombre de determinadas bases de datos seleccionadas previamente por los editores de esta red. También fueron observados otros posibles criterios selectivos, entre ellos el nombre de la base de datos, el precio de consulta y el formato de sus registros.

4 OBERMAN, C.: "Avoiding the Cereal Syndrome, or Critical thinking in the Electronic Environment", Library Trends, 39 (3), 1991, p.190

5 WIBERLEY Y DAUGHERTY (1988) op.cit. en OBERMAN, C (1991) op. cit. pp. 194-195.

6 MEYER, D.E. y RUIZ, D.: "End-user selection of databases. Part IV: People / News / General Reference", Database, 13 (5), 1990, pp.65-67 
Meyer y Ruiz partían de la hipótesis de que los usuarios evitarían consultar aquellas bases de datos que tuvieran el símbolo de recargo económico. Asimismo, pensaron que los archivos con nombres que describían más claramente los contenidos de sus bases de datos serían preferentemente consultados frente a aquéllos con nombres ambiguos o poco familiares. Por último, los autores también predecían que los usuarios preferirían la consulta de las bases de datos que ofrecían sus registros, primero, en formato de texto completo, y segundo, en formato resumen, mientras que las menos consultadas serían aquéllas que ofrecían sus registros en formato referencia.

Los resultados hallados no demostraron la influencia de ninguno de estos tres últimos indicadores. Sin embargo, no ocurrió lo mismo con el "Indicador de recomendación". En este caso, los resultados fueron altamente significativos. La mayoría de los usuarios se decidía por la consulta de aquellas bases de datos que eran recomendadas por los editores, independientemente del coste económico de las mismas, de si el nombre que las designaba perfilaba bien su contenido o no, o del formato de sus registros.

Ello demuestra a las claras la necesidad de organizar, clasificar y establecer un orden de preferencia (en función del contenido temático y de su grado de especialización) entre las incontables bases de datos existentes en la actualidad. Una clasificación y un orden que deben ser asignados tanto por los editores de las mismas, para ayudar a los buscadores de información en su difícil toma de decisión acerca de qué bases de datos han de consultar preferiblemente, como también por el propio usuario, cuya actitud crítica le debe permitir decidir con seguridad cuáles de esas bases recomendadas responden más claramente a su perfil particular de búsqueda.

Este mismo fenómeno de explosión informativa, aunque, si cabe, a mayor escala, es el que se ha producido a nivel mundial con la difusión o propagación de Internet, «el siguiente paso -como nos dice Kerckhove- en la evolución humana [...], con un efecto de primera magnitud comparable al del lenguaje escrito» ${ }^{7}$.

El Departamento de Defensa de los Estados Unidos sentó las bases de la futura Internet hace apenas treinta años con una red denominada ARPANET creada por la Agencia de Proyectos de Investigación Avanzados (ARPA). ARPANET sólo estaba al alcance de los profesionales de la informática, ingenieros y científicos que sabían moverse por sus complejos recovecos. Va a ser a partir de 1991, año del nacimiento de la World Wide Web desarrollada por Tim Berners-Lee y otros miembros del Laboratorio Europeo de Física de Partículas, cuando la navegación por Internet se convierta paulatinamente en un fenómeno social, pa-

7 KERCKHOVE, D.: «Internet es un nuevo paso en la evolución humana», entrevista en Muy interesante, $\mathrm{n}^{\circ} 229$ (junio), 130-133, 2000 
sando a formar parte de la vida cotidiana de millones de internautas de todo el planeta $^{8}$. Actualmente la World Wide Web alberga millones de sitios Web, creados por empresas, agencias, instituciones, personas, etc.

Es indudable que quienes dedicamos nuestra vida profesional o investigadora al ámbito de la Información y de la Comunicación debemos conocer, asimilar -y dominar- con la mayor prontitud posible este importantísimo cambio tecnológico, esta revolución social que ha supuesto Internet. Hoy día, como todos sabemos, ya no es necesario esperar al periódico de la mañana para saber lo que sucede en el mundo. Muchos sitios web dedicados a la información se actualizan varias veces a lo largo del día, procurándonos noticias políticas, nacionales o internacionales, económicas, deportivas, culturales, meteorológicas, etc.

La primera vez que navegamos por Internet podemos sufrir la sensación de estar realizando un safari por la sabana africana, a la caza del león -o de la información en este caso-, sin guía experto que nos ayude a adentrarnos, sin temor a perdernos, por esas tierras para nosotros extrañas y desconocidas.

De todas maneras, a falta de un guía personal con que contar, para navegar por Internet se han ido diseñando y perfeccionando herramientas, como los buscadores o motores de búsqueda y directorios que, a modo de mapas, nos facilitan enormemente la búsqueda, al revisar, valorar, organizar y crear índices de las informaciones que fluyen por los inmumerables nodos web que existen en la actualidad, aunque cierto es que ningún motor de búsqueda tiene un registro completo de los contenidos de Internet. Hasta los mayores motores de búsqueda, como Altavista (http://www.altavista.magallanes.net), Excite (http:// www.excite.com), Infoseek (http://www.infoseek.com), Lycos (http:// www.lycos.com), Yahoo (http://www.yahoo.com), etc, son incapaces de proporcionar toda la información que circula por esta red de redes.

En lo referente a los directorios existentes sobre Comunicación ${ }^{9}$, debemos hacer especial mención al directorio de Ciencias de la Información de la Universidad Complutense de Madrid (http://www.ucm.es/BUCM/inf/0500.htm), al índice sobre servicios de comunicación, Mediapolis. The media guide, elaborado por una empresa catalana (http://mediapolis.es/) y al Servidor de Estudios Cinematográfi$\cos$ y Audiovisuales (http://www.imaginet.fr/secav/adc/) ideado por la empresa Imaginet, que incluye varios directorios relacionados con el mundo audiovisual.

8 Algunas páginas que podemos consultar para conocer la historia de Internet y de la WWW son: www.cybernet.com.mx/hisint.html - www.w3.org/History.html

9 Para un conocimiento específico de los recursos de Internet en Información y Comunicación, recomendamos la lectura del capítulo "La Comunicación en Internet" (pp.417-449), de la autora Delgado Soler. En GARCÍA GUTIÉRREZ, A. (ed.): Introducción a la Documentación Informativa y Periodística, Sevilla: MAD, 1999. 
Al igual que ha ocurrido con los bancos y bases de datos, el notable incremento en el número de motores de búsqueda ha dado lugar en la red a la emergencia de nuevas herramientas altamente útiles y prácticas. Nos referimos a los metabuscadores, en especial a los de búsqueda conjunta, caso de Metacrawler (http://www.metacrawler.com) o Search (http://www.search.com), páginas web que lanzan la pregunta formulada por el usuario a varios motores de búsqueda al mismo tiempo, haciendo que el acceso a la información sea más rápido, cómodo y sencillo. El empleo de metabuscadores resulta más lento que si utilizamos un solo motor de búsqueda, pero la probabilidad de conseguir un resultado más completo es lógicamente mayor.

En la mayoría de las bases de datos, de los motores de búsqueda y de los metabuscadores la búsqueda de información se efectúa de igual manera, es decir, bien utilizando unitérminos o conceptos aislados (por ejemplo, politica) o bien combinando dos o más términos mediante operadores lógicos, lo que permite especificar nuestra demanda de información. Dichos operadores lógicos son básicamente:

- AND (en algunos motores de búsqueda, caso de Altavista, se emplea, en su lugar, el signo "+", mientras en otros se sobreentiende la conjunción entre los términos sin necesidad de especificarlo): equivale a la conjunción "Y" e indica que han de aparecer en el mismo documento todos los términos que especificamos restringiendo, de este modo, la búsqueda, por ejemplo: política AND europa (politica + europa).

- OR: equivale a la conjunción "O" e indica que han de aparecer en el documento indistintamente uno u otro de los términos que especificamos, ampliando la búsqueda, por ejemplo: Europa AND (politica OR gobierno).

- NOT: (en algunos motores de búsqueda, caso de Altavista, se emplea, en su lugar, el signo "-"): equivale al adverbio "NO" e indica que sólo se recuperarán aquellos documentos en los que figure el primer término especificado pero nunca el segundo, esto es, excluye al segundo término. Por ejemplo, europa NOT francia (europa-francia).

Otras fórmulas útiles de búsqueda de información serían las siguientes:

- COMILlAS: Para buscar una frase exacta, ésta habrá de encerrarse entre comillas: "politica agraria europea".

- ASTERISCO: Para buscar las diferentes formas de una palabra se agregará un asterisco (*) que sustituye a cualquier cadena de caracteres (en Lycos el asterisco se sustituye por el símbolo del dólar). Por ejemplo, si realizamos la búsqueda document* (document $\$$ ), se recuperarán todos los documentos que contengan alguna palabra con dicha raíz: document-acion, document-ar, document-al, document-ales, document-alista, etc. 
- INTERROGACIÓN: sustituye sólo a un carácter, normalmente, al final del término, aunque también puede colocarse en medio de una palabra. Así, al realizar la búsqueda politic?, se recuperarán los documentos que contengan la palabra politic-a o politic-o. Si utilizamos un término de búsqueda como $c a$ ?a: obtendremos como resultado documentos donde figuran palabras como casa, caza, cata, cala, etc.

- PUNTO: se utiliza (especialmente en el motor de búsqueda Lycos) para establecer los límites exactos de un término, por ejemplo: documental. no recuperándose términos de la misma raíz, caso de documentales, documentalista, etc.

Los patrones de búsqueda aquí vistos nos ayudarán y facilitarán la tarea de hallar información en las incontables bases de datos y entre los miles de nodos webs existentes en la actualidad. De hecho, y a título meramente indicativo, podemos decir que mientras que en junio de 1993 la web sólo abarcaba 130 sitios, un año después el número había crecido hasta casi 3000. En 1998 ya se contaba con más de 2,2 millones de sitios en la web.

En definitiva, y frente a toda la maraña de información con la que nos enfrentamos cada vez que investigamos, nuestra única salida es saber buscar, es decir, conocer y dominar la estrategia de búsqueda documental, una estrategia que podría quedar someramente representada en la Figura 1. Podemos afirmar que los tres requisitos indispensables para saber -y poder- buscar información eficazmente son:

1. En primer lugar, conocer qué tipos de fuentes de información existen, puesto que al profesional de la comunicación no le basta -o no le ha de bastar- con tener un archivo de recortes de noticias (fuentes periodísticas), por muy completo que éste sea. Aunque resulta difícil establecer una tipología universalmente aceptada de las fuentes de información existentes en la actualidad, dada la gran diversidad de ellas, una clasificación general de las mismas -en función de la procedencia y origen de la información- podría ser la que sigue:

a) Fuentes directas de información (personales u organizacionales), siendo la forma de transmisión del conocimiento normalmente oral (por medio de la entrevista, de ruedas de prensa, de charlas en actos públicos, etc.), aunque en la actualidad también es posible acceder por Internet a las informaciones proporcionadas por organismos públicos, como pueda ser la Junta de Andalucía (http://www.junta-andalucia.es/indice.org/).

b) Fuentes documentales, de transmisión escrita, dentro de las cuales podemos destacar esencialmente las siguientes: 
- Obras de referencia de información directa: diccionarios, directorios y guías; o de información indirecta: catálogos, boletines de sumarios, índices de cita, repertorios bibliográficos analíticos; estos últimos de gran utilidad, ofrecen, además de una referencia bibliográfica, un resumen del contenido del documento. Se trata de bibliografías especializadas, que suelen cubrir todo lo publicado en el mundo acerca de una determinada área temática o disciplinar.

- Fuentes primarias: manuales, compilaciones, monografías. La Base de Datos de Bibliografía Española en CD-ROM nos permite consultar, por multitud de campos combinables, los libros publicados en nuestro país desde 1976 hasta nuestros días (la base de datos se actualiza trimestralmente). Asimismo, para localizar fuentes bibliográficas españolas pueden consultarse las siguientes direcciones electrónicas: http://www.mcu.es/bases/spa/isbn/ ISBN.html. (Agencia Española de ISBN); http://www.direct.com/editores/ director.htm (Guía de Editores de España); http://www.mcu.es/bpe/bpe.html (Documentos de las bibliotecas públicas); http://eubd1.ugr.es/temp/serbydor/ home2.htm (página que nos permite acceder a bibliotecas universitarias españolas y de todo el mundo).

- Publicaciones periódicas (revistas y diarios), muchas de las cuales pueden ser consultadas hoy en día electrónicamente, caso por ejemplo -entre otros muchos- de los siguientes diarios: http://www.el-mundo.es (El Mundo); http://.elpais.es (El País); http://diario-vasco.com (El Diario Vasco); http:// www2.vanguardia.es (La Vanguardia); http://www.telebase.es/lavoz (La Voz de Almería); http://www.otd.es/infor (Huelva Información).

- Documentos audiovisuales (videográficos y fílmicos), sonoros y fotográficos. También la mayoría de las organizaciones dedicadas a la producción de material audiovisual y sonoro se han sumado a la red. Entre las muchas emisoras y televisiones que cuentan con páginas web destacaremos las siguientes: http://www.cadenaser.es; http://www.cope.es; http://www.rtve.es/ rne/index.htm; http://www.ondacero.es.; http://www.telecinco.es http:// www.rtve.es; http://www.canalsur.es; http://antena3tv.es; http://www.cplus.es.

- Literatura gris. Buen ejemplo de «literatura gris" son las tesis doctorales. Aquellas aprobadas en universidades españolas desde 1976 pueden consultarse en la Base de Datos TESEO (se actualiza mensualmente), en la siguiente dirección electrónica: http://www.mec.es/teseo/.

De todas estas fuentes citadas, el buscador habrá de decidir cuáles le interesa manejar y, a su vez, a cuáles le interesa acceder, en función de que resulten más o menos apropiadas e idóneas para obtener, en cada caso, la información que precisa. 
2. En segundo lugar, para saber dónde localizar dichas fuentes de información es necesario a su vez conocer los diversos centros de documentación existentes en el entorno en que nos movemos, y cuáles son los servicios que nos proporcionan. Valle Gastaminza ${ }^{10}$ nos aporta una tipología sencilla y útil de tales centros:

a) Centros cuya función es la conservación, tratamiento y difusión de documentos primarios o fuentes originales. Ese es el caso de los archivos, bibliotecas, hemerotecas, fototecas, videotecas, fonotecas y filmotecas. Muchos de estos centros ya pueden consultarse electrónicamente. Así, por ejemplo: http://www.mcu.es/lab/archivos/index.html (Directorio de Archivos de España) http://www.mcu.es/cine/index.html (Filmoteca Española. Ministerio de Cultura); http://www.cica.es/filmo/ (Filmoteca de Andalucía); http://www.sefes.es/index/index (Archivo Fotográfico INDEX).

b) Centros cuya función es el tratamiento y difusión de referencias (mensajes documentales). Cabe considerar dentro de esta categoría los centros de documentación científica general, los centros de documentación científica especializada y los centros de documentación de empresas u organismos privados (como puedan ser los medios de comunicación). Entre los muchos centros que pueden ser visitados a través de Internet, mencionaremos, a modo ilustrativo, dos direcciones electrónicas de interés para los profesionales de la información y la comunicación: http://www.csic.es/ (Consejo Superior de Investigaciones Científicas); http://www.cem.itesm.mx/dacs/ coneicc/ (Consejo Nacional para la Enseñanza y la Investigación de las Ciencias de la Comunicación).

3. Y en tercer lugar, una vez conseguidos los documentos que nos son útiles, debemos saber tratarlos adecuadamente para aprovechar de ellos la información esencial. Para lograr este objetivo, debemos dominar los procesos y técnicas documentales que nos ayudan a determinar el mejor modo de clasificarlos, resumirlos (bien realizando un resumen informativo, indicativo o analítico-descriptivo, según la importancia que concedamos al contenido del documento) e indizarlos (extraer de ellos las palabras claves), evitando los problemas derivados de una incontrolada sinonimia o polisemia que impidan una recuperación efectiva de los documentos archivados. De este modo, iremos

10 VAlle GASTAMiNZA, F. del: «Las funciones documentales: Tipología de centros y servicios de documentación». En LÓPEZ YEPES, J. (Comp.): Fundamentos de Información y Documentación, Madrid: EUDEMA, 1990.- pp.210-215 
construyendo un efectivo archivo personal, en el cual los documentos que hemos ido hallando estarán perfectamente organizados y tratados, sirviéndonos así no sólo en el momento presente en el que nos urge su utilización -motivo por el que iniciamos su búsqueda-, sino también en el futuro, en caso de que volvamos a necesitarlos.

\section{Conclusiones}

Todos los conocimientos aquí expuestos podemos adquirirlos por dos vías: a través de nuestra experiencia personal, con el gasto de tiempo y recursos (económicos, intelectuales, etc) que ello supone, o a través del estudio de las distintas especialidades de la Documentación relacionadas con el mundo de la comunicación (Documentación Periodística, Documentación Audiovisual, Documentación Fotográfica, Documentación Sonora, etc.), que proporcionan, cada una en su propio terreno, las técnicas y herramientas básicas para encaminar con éxito nuestras búsquedas de información. Es imprescindible que el nuevo profesional de la comunicación reciba una buena formación documental, la cual le ayudará a superar sus lagunas acerca de la búsqueda y la recuperación de los documentos que requiere.

Por todo ello es por lo que la Documentación debe dejar de verse o considerarse por nosotros mismos, los profesionales de la información, como una disciplina alejada de las Ciencias de la Información. A través de las distintas materias especializadas que se cursan en esta licenciatura (Documentación Audiovisual, Documentación Periodística...) se intenta acercar a los futuros profesionales de la información a las técnicas documentales propias de nuestra rama del saber, técnicas cuyo conocimiento y dominio podrán servirles no sólo como posible vía de salida profesional -trabajando en servicios de documentación de medios de comunicación-, sino también como instrumento básico de su trabajo como periodistas, para quienes conocer los cauces de información y saber analizarlos es algo elemental. La Documentación es un proceso que ayuda a activar nuestra actitud crítica, a resolver nuestros problemas como buscadores de información y, en definitiva, a entender, controlar y actuar sobre el universo de mensajes en que nos movemos y que constituye nuestro entorno vital. 


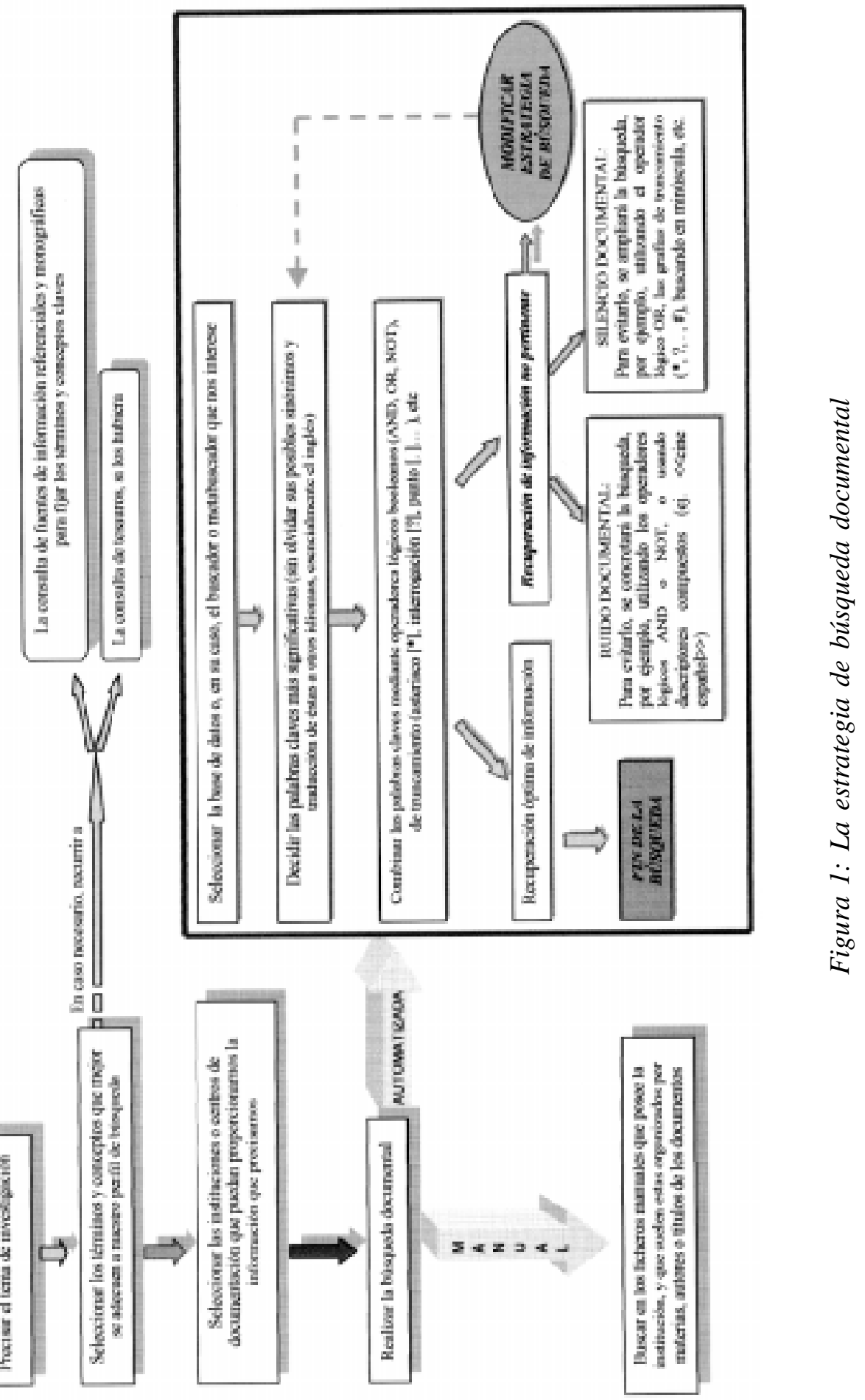




\section{Bibliografía:}

- CARRIZO, G. et al.: Manual de Fuentes de Información, Madrid: Cegal, 1994

- CODINA, L.: "Cómo funcionan los servicios de búsqueda en Internet: un informe especial para navegantes y creadores de Información (Parte I), Information World en Español, mayo 1997, vol. 6, n 6, pp. 22-27

- CODINA, L: "Cómo funcionan los servicios de búsqueda en Internet: un informe especial para navegantes y creadores de Información (Parte II), Information World en Español, junio 1997, vol. 6, $\mathrm{n}^{\circ}$ 6, pp. 18-27

- CORDÓN GARCÍA, J. A.; LÓPEZ LUCAS, J.; VAQUERO PULIDO, J. R.: Manual de búsqueda documental y práctica bibliográfica, Madrid: Pirámide, 1999

- DANIEL, E.H.: "Quality Control of Documents", Library Trends, 41 (4), 1993, pp. 644-664.

- DÍAZ NOCI, J.; MESO AYERDI, K.: Medios de comunicación en Internet, Madrid: Anaya Multimedia. Guía de navegación, 1997.

- GARCÍA GUTIÉRREZ, A.: Introducción a la Documentación Informativa y Periodística, Sevilla: MAD, 1999

- KERCKHOVE, D.: «Internet es un nuevo paso en la evolución humana», entrevista en Muy interesante, $\mathrm{n}^{\circ} 229$ (junio), 130-133, 2000

- LÓPEZ YEPES, J.: Fundamentos de Información y Documentación, Madrid: EUDEMA, 1990 ( $2^{\mathrm{a}}$ edic.)

- LÓPEZ YEPES, J.: Los caminos de la información. Cómo buscar, seleccionar y organizar las fuentes de nuestra documentación personal, Madrid: Editorial Fragua, 1997

- MARTÍNEZ LÓPEZ, F. J. et al.: Internet para investigadores: relación y localización de recursos en la red para investigadores y universitarios, Huelva: Servicio de Publicaciones de la Universidad, 1997

- MEYER, D.E. y RUIZ, D.: "End-user selection of databases. Part IV: People / News / General Reference", Database, 13 (5), 1990, pp.65-67

- OBERMAN, C.: "Avoiding the Cereal Syndrome, or Critical thinking in the Electronic Environment", Library Trends, 39 (3), 1991, pp.189-202

- SABOR, J.E.: Manual de Fuentes de Información, Buenos Aires: Marymar, 1978 ( $3^{\text {a }}$ edic.)

- TORRES RAMÍREZ, I.: Las fuentes de información. Estudios teórico-prácticos, Madrid: Síntesis, 1998 